\title{
Shape Properties and Perception
}

\author{
Kirk Ludwig
}

\section{Introduction}

We can perceive shapes visually and tactilely, and the information we gain about shapes through both sensory modalities is integrated smoothly into and functions in the same way in our behavior independently of whether we gain it by sight or touch. There seems to be no reason in principle we couldn't perceive shapes through other sensory modalities as well, although as a matter of fact we do not. While we can identify shapes through other sensory modalities -e.g., I may know by smell (the scent of mango) that the object causing my sensory experience is round - this is not perceiving an object as shaped, but rather inferring from the character of one's sensory experience and collateral information that an object of a certain shape caused it. That it is possible to perceive shape through other sensory modalities, however, is suggested by the case of bats and aquatic mammals like dolphins which navigate through their environment by a form of sonar. It is plausible that they have some form of auditory representation of space, and so of shape. These facts about shape perception raise important questions about the relation between those features of perceptual experience which are intrinsic to different sensory modalities and the nature of our per- 
ceptual representation of shapes, and, more generally, of the space within which we perceive shaped objects to be located.

John Campbell's paper, "Molyneux's Problem" (see above), raises a number of interesting and important questions about the nature of our perception of shape properties, particularly the cross-modal nature of shape perception, and ties them to more general questions about the nature both of perceptual content - whether it should be understood externalistically - and of shape properties - whether they should be understood categorically or as analyzable ultimately in terms of dispositions of objects. The question Campbell starts with is

Q1. "whether there is a difference between the phenomenal characters of shape experience in sight and touch" (p. 301).

This is one of the questions prompted by Molyneux's Question:

Suppose a man born blind, and now adult, and taught by his touch to distinguish between a cube and a sphere of the same metal, and nighly of the same bigness, so as to tell, when he felt one and the other, which is the cube and which is the sphere. Suppose then the cube and the sphere placed on a table, and the blind man be made to see: Quaere: whether by his sight, before he touched them, could he distinguish and tell which was the globe and which the cube? (Locke 1975, II, ix, 8)

If there is no phenomenal difference between perception of shape in sight and touch, then a man blind from birth whose sight is fully restored as an adult should, it seems, have no trouble visually distinguishing the globe from the cube. ${ }^{1}$ Clearly, the nature of perceptual representation of shapes is important to answering Q1, and, in particular, whether perceptual representation of shapes is constructed in some way out of different phenomenal or subjective features in tactile and visual sensory experience.

While Campbell does not explicitly provide an answer to Q1, I believe the answer he favors is that there is no difference between the phenomenal characters of shape experience in sight and touch. However, the main aim of Campbell's paper is not to provide and argue for an answer to Q1, but rather to connect its answer to the answers to a number of other questions about the nature of perceptual experience of shape properties. The first of these is,

${ }^{1}$ I raise some doubts in section 5 about the importance of there being no phenomenal difference between visual and tactile experience of shape for whether someone can know that he sees the same shape that he feels. 


\section{Q2. Ought we to be externalists ${ }^{2}$ about shape perception?}

Again, Campbell does not explicitly argue for externalism about perceptual representation of shape, but it seems clear that he is sympathetic to it. This question is in turn linked to the question,

\section{Q3. Are shape properties categorical properties or not?}

We will explore more fully below what being categorical comes to, but in the first instance the contrast is with properties that are dispositional properties or conceptually analyzable in terms of dispositional properties. It is not clear that Campbell gives a definite answer to this question either, though it seems that he is in sympathy with the view that shape properties are categorical properties. The two issues raised by Q2 and Q3 are in turn linked to the answer to another question, namely,

\section{Q4. Is the cross-modal transfer of information about shape rational} or not?

Cross-modal transfer of information about shape is the coordination of perception of shapes through different modalities in the production of behavior, in the sense that the information gained is integrated into and functions in the same way in the production of behavior independently of the sensory modality through which it is received. The cross-modal transfer of information is rational iff it is the result of the subject's access to the representation of shape in the experience itself. The alternative is that the cross-modal transfer of information is simply hardwired into us, a brute fact about our functional organization. On this view, there would be a physical explanation of it, and perhaps an evolutionary one, but no explanation which involved claims about the subject's recognizing that his perceptual experience was itself of an object of a certain shape. If we assume, as I think we should, that if something is in the content of one's perceptual experience, it is accessible to one in consciousness, then the

\footnotetext{
${ }^{2}$ Campbell distinguishes between radical and modest externalism about shape perception. It is not clear to me how to understand 'modest externalism', but since Campbell's concern is clearly with what he calls 'radical externalism', I will concentrate on that. Radical externalism appears to be the view that the representational elements of perception of shape are entirely determined by relational properties of the perceiver, and, in particular, relations to objects possessing the properties the individual represents. (What I mean by 'relational property' is the following: A property $P$ is a relational property iff $\mathrm{df}_{\text {necessarily, for all } x, x \text { has }}$ $P$ iff there is a $y$ such that $y \neq x$ or any part of $x$ and $y$ is a contingent existent.) Henceforth, by 'externalism', I will mean this view.
} 
second view reduces to the view that one's perceptual experiences of shape do not contain any representations of shape. The beliefs that would arise from perceptual experience about shapes would not, then, have their contents derived from the contents of the perceptual experiences; rather, certain perceptual experiences that do not represent shapes would cause in us beliefs about shapes. ${ }^{3}$ It is unclear to me, however, whether Campbell accepts the assumption on which this reduction depends. Again, while Campbell does not say outright that he thinks that the cross-modal transfer of information about shape is rational, he seems to be in sympathy with the view that it is.

These questions give rise to a fourth question, which I believe is the main focus of Campbell's paper, namely,

Q5. What is the relation between the answers to questions Q1-Q4?

It is not as clear as it could be how Campbell wants to answer this question, but I think at least the following theses can be discerned.

[T1] If shape properties are categorical, then perception of shapes is externalist.

[T2] If perception of shape properties is externalist, then there is no difference between the phenomenal character of shape experience in sight and touch.

[T3] If externalism about shape perception is correct, then crossmodal transfer of information is rational.

The path to these conclusions is complicated, and reconstruction of arguments for them requires some detective work. I am by no means confident that I have identified the arguments or theses correctly. But these theses, if correct, are important results, particularly [T2], which would establish a surprising connection between the categoricity of shape properties and how perceptual representation of shape is determined.

With respect to the primary questions, Q1-Q4, my own view is that there is a phenomenal difference between perception of shapes

\footnotetext{
${ }^{3}$ This also raises a question about whether what would occur in this case would count as perceiving shape, as opposed to merely identifying it through sight or touch, as, in our example above, we might, through smell, identify an object as round. If there is this connection between perception of an object's having a property and the object's being represented in the experience as having it, then there is cross-modal transfer of information in perception only if it is rational. I will leave aside this worry for the rest of the discussion.
} 
in sight and touch, ${ }^{4}$ that externalism about perception of shape is incorrect, that shape properties are categorical, and that the crossmodal transfer of information in normal adult human beings is rational. With respect to the relations among the answers to these questions, I think that they are by and large independent of one another, and, in particular, that none of [T1]-[T3] can be supported by showing that the antecedent entails the consequent. I will not argue in this paper that there is a phenomenal difference between perception of shape in sight and touch, or that externalism about shape perception is false. I will argue that shape properties are categorical, and that the cross-modal transfer of information is rational. In part, my aim here is to show that the questions are most straightforwardly answered independently of the issues Campbell links them to. Finally, I will argue that Campbell has given us no reason to think that there is an entailment between the antecedents of [T1]-[T3] and their consequents.

The plan of the paper is as follows. In section 2, I consider question Q3, whether shape properties are categorical. In section 3, I take up question Q4, whether cross-modal transfer of information is rational. In section 4, I take up the relation between the categoricity of shape properties and internalism and externalism [T1]. That there is an important connection must be a major thesis of Campbell's paper, for roughly half of the paper is taken up with a discussion of the nature of shape properties. But I can find no interesting connection between the question whether shape properties are categorical or not and the question whether perceptual content of shape properties is externally determined. In section 5, I take up, briefly, [T2] and [T3], and conclude in section 6 . Each of sections $2-5$ can be read independently of the others. Thus, e.g., a reader primarily interested in the discussion of Campbell's arguments for [T2] could skip straight to section 4 .

\footnotetext{
${ }^{4}$ See (Martin 1992) for an interesting recent discussion of what this difference might come to. Martin argues that it is not, as O'Shaughnessy (1989) has urged, that there is no sense field in tactile experience of the sort there is in visual experience, but rather that visual experience is "experience of objects external to one as arranged in physical space," while tactile experience is "experience of objects as they come into contact with one's body"; he continues,

one is aware of one's body and its limits and so aware of objects coming into contact with one's body as they discernibly affect those limits. Normal visual experience is essentially experience of objects as they fall within the visual field; tactual experience is essentially experience of objects as they press from the outside onto the limits of a felt sensory field. (p. 210)
} 


\section{Are Shape Properties Categorical or Not?}

Categorical properties are contrasted with powers, that is, with dispositional properties, or properties which are definable by appeal to only logic and powers. The mark of a power, according to Campbell, is that it can be defined by the following formula,

[P] $X$ has $P$ iff if circumstances $C$ obtain, then $X$ responds in way $R$

(where the specification of way $R$ does not make appeal to the property $P$ being defined). In addition to powers, Campbell says there are conditional powers, which can be defined as follows,

[CP] $X$ has $P$ iff if $X$ has properties $Q_{1}, \ldots, Q_{n}$, then in circumstances $C, X$ responds in way $R$.

Campbell goes on to entertain the suggestion that shape properties might be, not powers, or conditional powers, but clusters of conditional powers, i.e., that shapes can be defined by the following form, where the $\left[\mathrm{CP}_{i}\right\rceil$ stand for conditional powers:

[CL] $X$ has $P$ iff $X$ has $\mathrm{CP}_{1}, \ldots, \mathrm{CP}_{n}$.

Campbell identifies this as Shoemaker's proposal (1984 pp. 206233) that all properties of spatio-temporal objects are clusters of powers as applied to shape properties in particular. This is not, I think, exactly right. The most important divergence between the way Campbell lays out the view he wishes to examine and Shoemaker's more general thesis is that Shoemaker's thesis is about what we might call the 'metaphysical' nature of properties, and is not about how our concepts of properties are analyzed. ${ }^{5}$ Since Campbell characterizes the view he is concerned with as being about how to analyze the concept of a shape property, it is not Shoemaker's view as applied to shape properties. The divergence is important, because the proper method for deciding issues about conceptual and metaphysical necessity are (thought to be) different. It is not, however, crucial to Campbell's line of argument that the view he considers be

${ }^{5}$ I am skeptical that there are any so-called metaphysical necessities which are not reducible to conceptual necessities. The examples which motivate the distinction, such as the putatively a posteriori discovery of the necessary truth that water consists of aggregates of $\mathrm{H}_{2} \mathrm{O}$ (and its isotopes) in the liquid state, are better understood as examples of the discovery of what concept 'water' expresses rather than of a new kind of necessity not grounded in our concepts. But I cannot pursue this issue further here. 
Shoemaker's view. In what follows, the view I intend to be evaluating is the one presented in Campbell paper, whether or not it is Shoemaker's view. ${ }^{6}$ I take this view to hold that two properties are identical iff the concepts which pick them out are identical, and that the proper method for investigating the nature of properties is by analyzing the concepts which pick them out.

Before considering more closely the proposal that shape properties can be analyzed in the form [CL], it is worth asking whether conditional powers really are distinct from simple powers. It appears that they are not, for any conditional power defined as in [CP] can be recharacterized in as in [P]. To see this, suppose we have a conditional power defined as above. Now let circumstances $C^{*}=C$ and $X$ 's having properties $Q_{1}, \ldots, Q_{n}$. Then we have

\section{[RE] $X$ has $P$ iff if circumstances $C^{*}$ obtain, $X$ reacts in way $R$.}

Thus, we could simply take the suggestion that shapes are clusters of conditional powers as the suggestion that they are clusters of powers, and simplify the discussion.

To determine whether shape properties are categorical properties or not, we need to know more about what a categorical property is. Categorical properties are to be understood by what they are contrasted with. Categorical properties are properties that cannot be defined as in $[\mathrm{P}],[\mathrm{CP}]$, or [CL]. Thus, we do not have to worry about their roles in explanations to explain what they are (see section 3 of Campbell's paper). They will, however, have a special role to play in explanations, in virtue of categorical properties being the ground of dispositional ones (though there's no reason to suppose all

\footnotetext{
${ }^{6}$ Shoemaker also makes some terminological distinctions that Campbell does not observe, and which I will not observe either. For example, Shoemaker draws a distinction between 'is dispositional' and 'is a power'. The former he treats as a predicate of predicates; the latter of properties. It should be noted also that Shoemaker is concerned with what he calls genuine properties, as opposed to (mere) Cambridge properties; according to Shoemaker, not every predicate expresses a property. Part of the motivation of his account is to provide a way of distinguishing between genuine properties and mere Cambridge properties. Genuine properties, however, as Shoemaker conceives them, are only properties which are involved in (genuine) changes; a third class of properties, neither genuine nor mere Cambridge properties, are those of abstract objects such as numbers, e.g., being prime or being divisible by two. This last point in particular suggests, given the epistemological nature of Shoemaker's argument (see below), that his conclusion should really be recast as the claim that the only properties we could know objects to have are one's characterizable essentially in terms of powers, not that properties of spatio-temporal objects must be individuated by their causal powers.
} 
categorical properties ground dispositional powers). What is it for a categorical property to ground a dispositional one?

[G] $P$ is the categorical ground of a disposition to $R$ when $C$ iff $P$ is a categorical property and it is a causal law that if $X$ has $P$ and circumstances $C$ obtain, then $X$ responds in way $R$.

To say it is a causal law that if $X$ is $P$ and circumstances $C$ obtain, then $X$ responds in way $R$, is not to give a definition or conceptual analysis of the predicate 'has $P$ ', but to make a remark about the nomic role the property picked out has in physically possible worlds. This is a contingent fact, and things could have been otherwise, and it must be established by a posteriori investigation.

It is evident that there must be categorical properties, if there are dispositional ones. For, first, there must be properties which figure in the fundamental laws which explain why objects have the dispositions that they do. And, second, if every property were spelled out dispositionally, so that our understanding of the concept which picked out the property were expressed in a counterfactual conditional, then there would be no properties for which we had concepts. Powers are defined in terms of conditionals. The antecedents and consequents must employ predicates that express properties. The conditional has no content until we specify what those properties are. If for each of those predicates we had to substitute a conditional, and then for each predicate in the substituted conditional another conditional, ad infinitum, then clearly no properties would be fixed at all.

Now we are in a position to answer the question whether shape properties are categorical properties. It is sufficient (though perhaps not necessary) for a property not to be a categorical property that no biconditional of the form $[\mathrm{P}],[\mathrm{CP}]$, or $[\mathrm{CL}]$ be necessarily true with a predicate expressing the property in the place of 'has $P$ '. Representing the universally quantified biconditionals of these forms as predicates of properties as follows ' $[\mathrm{P}](p)$ ', ' $[\mathrm{CP}](p)$ ', and ' $[\mathrm{CL}](p)$ ', where $p$ is a variable for the property being characterized, we can put the condition as follows:

[C] $(p)(p$ is a categorical property provided that it is not necessary that $[\mathrm{P}](p)$ or $[\mathrm{CP}](p)$ or $[\mathrm{CL}](p))$.

Are shape properties then categorical? It will help to ask a bit more particularly about what we have in mind in talking about shape properties. When we talk about an object's having a shape, e.g., of an object's being spherical, we ascribe a property to an object located 
in space. Now consider the space which that object occupies. Can we speak of the shape of that region of space? Yes. For remember that we also represent the regions of space between and around the objects which we represent in it, and that this is essential to our representation of the shapes of those objects. But now in talking of the shapes of regions of space, we are not talking about an object in the usual sense, something which occupies space, and can move about within it, though we are clearly employing the same concepts as those we apply to objects. The two uses are of course connected: an object has a certain shape just in case the region of space it occupies (at a time) has the same shape.

Let's take 'shape' as applied to regions of space first. Since space is thought of as causally neutral, the stage where the play takes place, rather than a participant in the action, it is not clear that there are any biconditionals of the form (3) true as matter of fact of shape properties of regions of space, let alone necessarily true. Our concept of shape properties then cannot be captured by our concept of any cluster of powers. This is the central difficulty in trying to analyze shape properties in terms of powers. Powers are properties of objects. If they are objects which have shapes, they have spatial locations. Their shapes, however, are understood in terms of the geometry of the regions of the space in which they are located. Our conception of that space and its geometry is independent of that of the objects located within it. ${ }^{7}$ Thus, our understanding of the shape properties of objects could not be reduced to powers which they possess, since this leaves no room for an independent conception of the geometry of the space in which they are located and in terms of which their shapes are understood.

Now consider 'shape' as applied to objects. We understand what it is for an object to have a shape in terms of the shape of the region of space it occupies. Does the shape of an object necessarily have any consequences for what it will do in various circumstances? This depends, I think, more on our concept of an object than on our concept of shape. To see this, consider the contrast between rigid and non-rigid objects. A rigid object is one that retains its shape (and size) under all conditions (that is, the distance between any two points in it remains constant through all forces exerted upon it). A non-rigid object is one that does not. This is not a contingent fact about rigid objects; nothing counts as a rigid object of which

\footnotetext{
${ }^{7}$ This assumes, as I think correct, that spatial relations cannot be reduced to relations among objects; arguments to the contrary from a principle of sufficient reason or verificationism rest on doubtful premises.
} 
this is not true. (In point of fact, there are no rigid objects; rigid objects are an idealization in physics, like a frictionless plane.) A rigid and non-rigid object could have the same shape. But they will do different things in different circumstances. For example, the rigid square peg will not go through a hole in a rigid board whose diameter is the same as the length of the peg's sides; a non-rigid peg may well do so - e.g., a square peg made of clay. It is not the shape and size of the peg relative to that of the hole in the board that determines whether or not it will pass through it, but rather the dispositions of the peg and board to retain their shapes in various circumstances.

Since we understand the contrast between rigid and non-rigid objects in terms of their dispositions to retain or not retain their shapes and sizes in various circumstances, and this distinction is crucial to saying what an object of a certain shape will do in various circumstances, it is clear that shape properties cannot be defined in terms of clusters of powers of objects. For to try to understand it that way, we would have to appeal to the concept of rigidity, which presupposes a prior grasp of the concept of shape.

The problem becomes painfully obvious when we consider inserting a characterization of rigidity into a characterization of shape in terms of rigidity.

[RI] $X$ is rigid iff for any shape $S$, and Size $Z$, if $X$ has $S$ and $Z$, then no matter what the circumstances $C, X$ has $S$ and $Z$.

Consider now how one might try to turn this around into a partial definition of shape in terms of what an object will do if it is rigid:

[RV] $X$ has $S$ only if, if $X$ is rigid, then no matter what the circumstances, $X$ has $S$.

There are really two problems here. First, of course, we have seen that 'rigid' is defined in terms of shape. Second, even apart from that, we see that the particular shape we are attempting to define here has to be mentioned in the consequent of the conditional. Clearly, no progress has been made.

Before leaving this section, we should take a brief look at two further matters. The first is the functional definition of shape properties which might be proposed to avoid the circularity problem (Campbell, p. 311). The second is Shoemaker's arguments, as represented by Campbell, that properties have to be understood as dispositional all the way down.

To get around the charge of circularity, Campbell suggests we can introduce the shape property by way of a description of it as the 
property which has a certain role in a theory of shape properties obtained in the following way. Consider a particular shape property $S$. Suppose it is definable as a cluster of conditional powers (or simply powers),

[T $\left.T_{0}\right](x)\left(x\right.$ has $S_{0}$ iff if $x$ has $Q$, then if $C, x$ will $R$ and if $x$ has $Q_{1}$, then if $C_{1}, x$ will $R_{1}$ and if $x$ has $Q_{2}$, then if $C_{2}, x$ will $R_{2}$

...

and if $x$ has $Q_{n}$, then if $C_{n}, x$ will $R_{n}$ ).

Note that in the $\left\lceil C_{i}\right\rceil$ s we will have to mention shape properties. Now, summarize the open sentence on the right hand side of the biconditional as ' $T_{0}\left(x, S_{0}, \ldots, S_{k}\right)$ ', where the $\left\lceil S_{i}\right\rceil$ represent the terms referring to shape properties in the specification of the circumstances. Now we represent the definition of $S_{0}$ as follows.

$\left[\operatorname{Def}_{c}-S_{0}\right](x)\left(x\right.$ has $S_{0}$ iff $\left.T_{0}\left(x, S_{0}, \ldots, S_{k}\right)\right)$.

This is a circular definition (since we will need to say how objects of the same shape interact in characterizing the dispositions of an object of any shape). Now, take the conjunction of all the true biconditionals of the form of [Def $\left.c_{c}-S_{0}\right]$, and represent this as a theory: [ $\left.T_{1}\right] T_{1}\left(S_{0}, \ldots, S_{r}\right)$.

Now replace each of the terms referring to shape properties with distinct variables and existentially quantify over each of the variables to get:

$\left[T_{2}\right]\left(\exists s_{0}\right)\left(\exists s_{1}\right) \ldots\left(\exists s_{r}\right) T_{1}\left(s_{0}, \ldots, s_{r}\right)$

This is the Ramsey sentence for $\left[T_{1}\right]$. Now, call the predicate obtained from $\left[T_{2}\right]$ by removing the existential quantifier over the $i$-th variable $\left\lceil T_{2}^{i}\left(s_{i}\right)\right\rceil$. Now, we can define each property $S_{i}$ as follows:

[Def- $\left.S_{i}\right](x)\left(x\right.$ has $S_{i}$ iff $x$ has $\left.\left(i s_{i}\right) T_{2}^{i}\left(s_{i}\right)\right){ }^{8}$

I want to note two points here. First, it is obvious that this is not how we understand shape properties, since there are an infinite number of shape properties, so that above the values of ' $k$ ' and ' $r$ ' will be infinite, and we clearly could not grasp the resulting proposition. Second, and quite apart from this, $\left[\operatorname{Def}-S_{i}\right]$ does not define $\left\lceil S_{i}\right]$ in terms of powers, for it is not strictly a definition. For all that [Def$\left.S_{i}\right]$ says $\left\lceil S_{i}\right\rceil$ could denote a categorical property. This is because

\footnotetext{
${ }^{8}$ Where as usual ' $\left(i s_{i}\right)$ ' is interpreted as 'the $s_{i}$ such that'.
} 
$\left[\right.$ Def- $\left.S_{i}\right]$ fixes the property picked out by $\left\lceil S_{i}\right\rceil$ by description. It is whatever unique property plays the appropriate role in the theory. That property, for all that has been said, may well be a categorical property. [Def- $S_{i}$ ] fixes the meaning of $\left\lceil\right.$ has $\left.S_{i}\right\rceil$ in the way one fixes the referent of a name by a description such as 'the first child born in the 21st century'. The description does not give the meaning. Thus, there is no reason to think that in fixing what property $\left\lceil S_{i}\right\rceil$ denotes in this way we have determined it to denote a property definable in terms of clusters of powers. Thus, this is not a response to the objection that definitions of shape properties in the form [CL] are going to be circular, and so fail as definitions. ${ }^{9}$ In conversation, Shoemaker has told me that this is not part of his aim, and that the content of his thesis is exhausted by the claim that the above biconditional is necessarily true.

Now let's turn to the arguments adapted by Campbell from Shoemaker. As I noted above, I think there are some important differences between the position that Campbell characterizes and Shoemaker's. I will be considering the arguments advanced below as they bear on the position that shape properties and properties in general are understandable in terms of powers as it is characterized by Campbell. It is clear that, in at least some cases, the arguments are not applicable to Shoemaker's own position.

The overall form of the argument is the following:

\footnotetext{
${ }^{9}$ I would like to insert a brief query about Shoemaker's own official proposal, which is represented as a criterion for individuating properties. Modified to meet an objection of Richard Boyd's (Shoemaker 1984, p. 233), it goes as follows,

$P_{1}=P_{2}$ iff necessarily, for all causal potentialities CP, $P_{1}$ has CP iff $P_{2}$ has CP and for all circumstances $C, C$ is sufficient to bring about a thing's having $P_{1}$ iff $C$ is sufficient to bring about a thing's having $P_{2}$.
}

Put this way, one can see that there is a striking parallel between Shoemaker's proposal for individuating properties and Davidson's for individuating events (Davidson 1980). Thus, it looks as if it suffers from the same defect Quine (1985 p. 166) pointed out in Davidson's attempt to individuate events in terms of their causes and effects. Since causal potentialities and circumstances quantified over on the right hand side of the biconditional themselves involve properties, that is, they are partly individuated in terms of what properties they involve, the right hand side of the biconditional above presupposes the individuation of properties, and so cannot explain in what their individuation consists. Whether this criticism applies to Shoemaker's account depends in part on what he means when he says that he is giving individuation conditions for properties. Quine's criticism presupposes that in giving individuation conditions for a kind of entity, the conditions one gives do not presuppose one already understands how to individuate the kind of entity in question. 
[A] 1. If properties are categorical, then we can never know that any object has them.

2. Whatever properties there are, we can know that some object has them.

3. Therefore, there are no categorical properties.

A first remark to make is that since there have to be categorical properties if there are powers, a further conclusion can be drawn from 3 , namely,

4. There are no properties, i.e., no object has any property.

Since there are objects only if they have properties, it follows,

5. There isn't anything. ${ }^{10}$

This is a conclusion sufficiently absurd to lead us to suppose that something has gone wrong with the argument for it.

First, there is no reason to think 2 is true. There may be many properties which objects have which we don't and can't know that they have because of the nature of the properties, or because of the nature of ourselves. Second, none of the reasons offered for 1 are any good. As represented by Campbell, these are:

[R1] If there are categorical properties, then it 'ought to be possible for there to be properties which make no difference whatever to the behaviour of the things which possess them.' (p. 311)

[R2] If there are categorical properties, then there 'could be different properties that make, under all possible circumstances, exactly the same contribution to the causal powers of the things that have them.' (p. 311)

[R3] If there are categorical properties, then the 'potential of a particular property for contributing to the production of causal powers might change over time.' (p. 311)

Consequently,

[C] '[W]e could have no knowledge of the properties of a thing since all we can know is the behaviour of the thing - and we would have no way of singling out a property in order to name it, and even if we did somehow manage to christen a property, there would be no way... in which we could know that we had encountered the same property again.' (p. 311)

\footnotetext{
${ }^{10} \mathrm{Or}$, rather, anything except abstract objects, in line with the qualification in footnote 6 .
} 
There is nothing in the concept of a categorical property which licenses any of [R1]-[R3]. What is required for properties to be categorical is that there be no biconditionals of the form $[\mathrm{CL}]$ (or $[\mathrm{P}]$ or $[\mathrm{CP}]$ ) which define them. With respect to [R1], it is compatible with this being so for shape properties that it is necessary that shape properties make a difference to the causal powers of the objects that have them, for that does not require that they make the same difference in every possible world. With respect to [R2], it is compatible with this being so that different shape properties necessarily make a different difference to an object's causal powers. With respect to [R3], it is compatible with this that shape properties necessarily make the same difference to an object's causal powers at every time. Furthermore, even if [R1]-[R3] were true, it is not clear that it would follow that we could have no knowledge of the properties of a thing. It is clear that the proponent of this argument supposes that we can have knowledge of dispositional properties, and since not all properties of objects can be inferred from antecedent knowledge of the objects' having other properties, on pain of an infinite regress, we must be capable of knowing for some properties and some objects that the objects have the properties without inferring that they do. The epistemic problem that the objector to categorical properties envisages rests on the assumption that we must infer that objects have categorical properties from the effects of their having those properties. Their effects will be manifested as properties of some objects that we can know the objects have non-inferentially. Clearly, to be consistent, the proponent of the argument must suppose those properties are powers or definable in terms of powers. Thus, the proponent of the current argument must hold that we can know directly that objects have dispositional properties, but that there is some special obstacle that prevents us from knowing directly that objects have categorical properties. But what is that obstacle? If we could know directly that objects have dispositoinal properties, why could we not know directly that objects have categorical properties? Indeed, the objector's assumption that we would have to infer what properties objects have from direct observation of (some at least of) their dispositional properties seems to get things upside down. If there is a priority of knowledge of the one sort of property over knowledge of the other sort, knowledge of the dispositional properties of objects should rest on knowledge of categorical properties of those objects. For we only know what powers a thing has by inductive reasoning. To discover a thing's powers, we must see what it does in various circumstances, i.e., we must have access to the manifestations of its powers. But these manifestations can't (ultimately) be themselves 
powers if our inductive reasoning is to have materials to work on to begin with. Thus, it seems that even if we grant [R1]-[R3], we have no reason to suppose we could not know that objects had categorical properties, and we have reason to think that unless we could know that objects had categorical properties, we could not know that they had dispositional properties or properties definable in terms of dispositional properties.

To summarize, we have seen that shape properties are categorical properties, that there is no response to the circularity objection in appeal to definition by way of Ramsey sentences, and that none of the arguments against shape properties being categorical properties we have examined are any good. Furthermore, our treatment of these issues has not required us to make any commitments about how the contents of our perceptual representations of shape are determined.

\section{Is Cross-Modal Transfer of Information Rational?}

We described the phenomenon of cross-modal transfer of information in a way that was neutral with respect to the two interpretations of it. Both of the interpretations are prima facie conceptually possible. In particular, it seems possible for the brute functional organization thesis to be true of some creatures who have concepts of shape. ${ }^{11}$ It also seems conceptually possible for perceptual experiences to contain in their contents representations of space and of shaped objects within that space (to which the perceiver has access). If both options are conceptually possible, then if we are to answer the question whether cross-modal transfer of information is rational, we cannot do so for all possible perceivers. We must relativize the question to particular groups of perceivers.

Clearly, the thesis that cross-modal transfer of information is rational, since it involves a claim about the content of perceptual experience and access to that content by the perceiver, can't be answered on the basis of behavioral evidence. The behavioral evidence will be compatible with either alternative. Therefore, where we can have no insight into the character of the experience from the point of view of its subject, we must be skeptics about the answer to the question.

\footnotetext{
${ }^{11}$ If there is some difficulty about this, it would have to be because of some important connection between the ability to possess the concept of space and the ability to experientially represent space. It is tempting to suppose that there is such a connection, but I cannot see how to make good on the claim.
} 


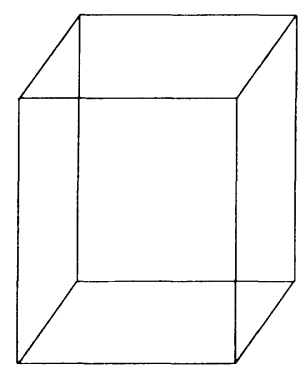

FIGURE 1. Necker cube.

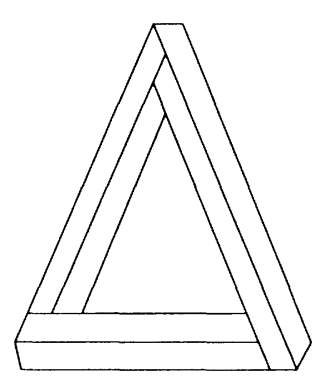

FIGURE 2. Penrose triangle.

This is plausibly the case with animals, and with infants and young (enough) children. I propose then to reformulate the question so that it is specifically about the phenomenon as it appears in normal adult human beings. Henceforth I will understand the question in this way.

Given that we have access to the contents of our conscious perceptual experiences, the question whether cross-modal transfer of information is rational comes down to the question whether we are aware of representations of shaped objects as part of our visual and tactile experiences of our environment. What is the answer to this question? I think we obviously do have access to such representational information in the content of our perceptual experiences. When I look out my window, I have a visual experience of, as I would put it, a number of heads, on a number of shoulders; that is a remark about the content of the experience, and in particular conveys some (not very precise) information about the shapes of objects which my visual experience represents. Likewise, as I hold this pen in my hand, I have a tactile experience of (and as of) a cylinder, as I would put it, which conveys information about the representational content of the tactile experience, and, in particular, about shapes of objects which it represents. As I look at the pen, my visual experience also represents it as cylindrical. These representational features of my experience are transparent to me, for my experiences phenomenally seem to put me in direct contact with the world that they represent, and I am representing a three dimensional spatial world containing objects of various shapes at various distances from me. It does no good to protest that this representational content is not part of the content of my perceptual experiences, but rather just of my beliefs, for even if I suspend belief about my environment, my perceptual experiences still represent it as being the same way. This is the possibility upon which hallucinations and illusions which persist through 
discovery depends, such as a 3-dimensional Necker cube (Figure 1), or Ames's distorted room (see Gregory, pp. 85-7), and the perceptual representation of objects recognized to be impossible (such as the Penrose triangle (Figure 2)). The question whether cross-modal transfer of information is rational in our case, then, seems to admit of a straightforward and incontrovertible answer: it is.

Before moving on, let us ask whether we find any reason to be skeptical of this result in the case of the man who is the subject of Molyneux's question, a man born blind, who can recognize by touch a sphere and a cube, whose sight is restored as an adult. Molyneux's question is whether he would be able to recognize by sight the sphere and the cube, that is, to know that the object which he sees is of the same shape as the object which he feels, when he sees the sphere, or cube, and when he touches it. How might the results of this experiment be used against the above claim? Suppose that the man could not at first recognize the object identified as the sphere by touch as that object by sight, but later comes to be able to do so. Then it might be urged that the connection is purely a functional one, since the content of his visual experience has not changed, and he could not originally make the identification, and surely at the end of the process his experience will be the same as ours.

There have been actual cases like the one imagined by Molyneux, in which an individual blind from birth because of opacity of the cornea, or because of cataracts, has had his sight restored by an operation. The empirical facts are not easy to interpret, however. In some of these cases, individuals required a long period of training to recognize and name even simple objects or shapes by sight. In others, they were able to see well almost immediately. The cases are difficult to interpret, because it is difficult to sort out by behavior cases in which physiological function has been restored completely from those in which it has been restored partially at first, and fully only slowly if at all. There does seem to be some suggestion that where the operation, as in cornea transplants, results immediately in a clear image on the retina, the individual is able almost immediately to recognize by sight objects which he is familiar with through touch. (See Gregory 1990, pp. 201ff.) However, whatever the empirical facts, the answer to Molyneux's question does not have the power to upset the conclusions above. The actual empirical facts are limited to whether or not the subject is able immediately to recognize shapes by sight or not, and then becomes able to do so later on. The objection considered above depends on making two further assumptions, which amount to an explanation of the empirical facts. The two assumptions, as we saw, are that the perceiver's experience 
does not change its character, and that his experience when he can identify shapes by sight is the same as ours. The empirical evidence is compatible with denying either of these. Thus, e.g., in coming to identify shapes as we do, our imagined subject may well have subjective experience of the sort we do, but as a result of the character of his perceptual experiences changing from the time when his sight was initially restored. This would be much the most likely explanation, in light of the fact that the representational content of visual experiences is a feature of its phenomenal content. Contrast auditory perception of an uttered sentence when one understands it and when one does not, or perception of script which one understands with perception of script which one does not. Or, again, consider the difference between different ways of seeing an ambiguous figure, such as the duck-rabbit, or a Necker cube. In all these cases, changes or differences in the representational content of the perceptual experience clearly affect what it is like to have them. Even if we did have good reason to think, however, that the subject's subjective experiences had not changed, there would still be explanations of the data compatible with the result reached above. Thus, e.g., we might think his experience was not in fact similar to ours, that in his case there was only a functional connection. It is hard to see, in fact, how the (third person) empirical evidence could overturn our first person authority over what the contents of our perceptual experiences are like. Therefore, even if a man blind from birth whose sight is restored would not be able identify the object he sees as the sphere he touches as opposed to the square that he touches, it would not show that cross-modal transfer of information is not rational in our case.

Thus, the answer to the question whether cross-modal transfer of information is rational is 'yes', and the answer can be given independently of any considerations that have to do with externalism or the nature of shape perception.

\section{How Are the Issues of the Categoricity of Shape Properties and Externalism about Perception of Shape Properties Related?}

It is not easy to see why Campbell thinks that the question whether shape properties are categorical is relevant to the question whether perceptual representation of shape properties is externalist. The question of the nature of shape properties enters into the discussion in the following passage: 
This gives one way of setting up the question of the sameness or difference of shape perception in sight and touch. But you might object that this is quite the wrong way in which to set up the problem. You might object that we have to think of shape properties in terms of their causal significance, and that shape perception is perception of this causal significance. In this section I will develop this causal approach to shape perception. Then I will argue that the approach is incomplete, and that there is still a crucial issue that is addressed by externalism about phenomenal experience of shape. (p. 304)

The 'sameness or difference of shape perception in sight and touch' refers here to whether there are phenomenal differences between perception of shape in sight and touch. It is not clear exactly what Campbell has in mind by 'this way of setting up the problem': the problem does not require setting up, it is expressed in a simple question, Q1. So it is mysterious what objection we are to imagine is being raised by the thought that shape properties are to be understood in terms of their causal significance (as clusters of powers (or conditional powers)). It is evident, though, from the last line of this passage, that Campbell thinks that this approach to understanding shape properties fails - 'is incomplete'- and that externalism is required in some way to address some 'crucial issue'. What is that crucial issue? How do we work our way around from a discussion of the nature of shape properties to whether the representation of those properties in perception is externally determined or not?

The transition takes place in two steps. First, we are invited to reflect on what perceiving shapes would come to on the view of shape properties as definable as clusters of conditional powers. Campbell represents this as a pressing need for the proponent of the cluster of powers view of shape properties:

On the face of it, we do not perceive the shape of a thing as a collection of unsubstantiated threats and promises as to which powers it will take on in various hypothetical circumstances. We perceive the substance behind the threats and promises. So we need some preliminary explanation of what it could mean to perceive an object as having a cluster of conditional powers, before we can understand the argument that that is all we perceive the object to have. ${ }^{12}$ (p. 306)

\footnotetext{
${ }^{12}$ Campbell does not explain how he is using the expression 'perceiving $x$ as ...', but I will take this to mean that the representational content of the perceptual state involved represents $x$ as falling under the concept expressed by what goes in for '...'.
} 
Campbell spends some time saying what it could mean, but the question and time spent are not well-motivated. What it means is as clear as the thesis that shape properties are definable as clusters of conditional powers. It is not a motivation for asking after the meaning of some claim that it is in conflict with common sense. At most that is a reason for inquiring after its truth. What it means is just that in perceiving something as having a certain shape, we perceive it as being such that in such and such conditions, it behaves in such and such ways, which conditions and ways depending on the shape in question. Of course, this has implications for our behavior, given that we are rational agents, as Campbell points out at length, but adding what those implications are, given our desires, does not shed any further light on what the claim is. In any case, this exercise sets up the next transition, which is the observation that if we want to deny that shape properties are wholly analyzable as clusters of conditional powers, then we must suppose that perception of shape properties involves "more" than perception of clusters of conditional powers.

On the alternative view, these connections [between having a shape and reacting in such and such ways in such and such conditions] do not exhaust the content of shape perception. In addition to these connections, shape perception provides knowledge of the shape property as the ground of all the functional patterns that are specified. The problem then is to explain just what this further knowledge provided by shape perception is, and to explain its relation to these further connections and patterns of functional role. (p. 312)

It should be noted that if shape properties are categorical properties, then it is understating the case to say that 'these connections do not exhaust the content of shape perception'. They are not part of the content of shape perception as such at all. But we may grant the point that if shape properties are categorical, one must accept that perception of shape properties is perception of categorical properties.

It is this point that apparently brings us back to the question of whether externalism about shape perception is correct. Campbell calls perception of shape as categorical 'primitive consciousness of shape'. (I cannot see that 'primitive consciousness of shape' comes to more than this.) Now, how do externalism and internalism enter into the picture? The transition takes place in the following passage:

The kind of primitive consciousness of shape I am describing is... extremely primitive. There would be none of the complex of functional connections to action and imagistic reasoning which I described earlier. 
That means that we have to consider the question what makes it so that this consciousness is consciousness of shapes. (p. 314)

It is to respond to the question posed in the last sentence here that Campbell turns to discuss externalism and internalism. The question is to be motivated by the first 2 sentences, tying it to the discussion of the nature of shape properties. It is difficult, however, to see the bearing of the first two sentences on the last. Primitive consciousness of shape just is perception of shapes as categorical, i.e., perception of shapes, if shape properties are categorical properties. Then of course it follows that such perception of shapes is not perception as such of clusters of conditional powers, and as such does not enter into deliberations about what to do in the way that perception of clusters of conditional powers would. Why does that mean that we have to consider the question "what makes it so that this consciousness is consciousness of shapes?"

In any case, internalism and externalism enter at this point. Externalism is supposed to provide the following answer to the question: since the "geometrical aspects of your experience of objects are constituted by the geometry of the objects in your surroundings," "... the character of experience as perception of shape can be secured without having to consider the relations of the perception to imagery and action" (p. 314). Internalism, too, is supposed to be prima facie in a position to answer the question: "an internalist account of shape experience, on which sensations are configured in a sensational space... would also suggest the possibility of a kind of primitive awareness of shape, on which it could be prior to the complex of functional connections to action and imagistic reasoning described above" (p. 314). However, it turns out that internalism faces a problem that externalism does not, and it seems that this is what provides finally a motivation for preferring externalism to internalism.

Before taking that up, however, we should consider whether up to this point we have been provided with a reason to think that the issue of whether shape properties are categorical is specially connected with the debate between internalism and externalism. The only reason provided appears to be the 'need' to raise the question what makes perception of shape as categorical perception of shape. Given the kind of answer offered to this question, it is clear that 'what makes it so' is being interpreted as 'what facts other than the fact that the perception is perception of shape are sufficient for it to be so'. I cannot see any reason why this question needs to be raised at all, or any reason to think that if it must be raised, it 
must be raised specifically in the case that shape properties are categorical. If it must be raised at all, why should it not need to be raised equally if shape properties are clusters of conditional powers? There is a hint of a reason in Campbell's paper in his discussion of what it would mean for perception of shapes to be perceptions of shapes as clusters of conditional powers. For it may be that Campbell thinks of that question as the same question as what makes it the case that perception of shape is perception of shape if shape properties are clusters of conditional powers. Then he may suppose he has given an answer independently of the internalism/externalism debate, so that that issue comes up only when we turn to the hypothesis that shape properties are categorical. But (i) the questions are not the same, and (ii) the answer Campbell earlier gives does not shed light on either of the questions, since the observation that we behave in ways appropriate to how we represent the world is not an answer to the question what it means to perceive shapes as clusters of conditional powers or to the question what makes representations of shapes representations of shapes. The question of the nature of shape properties, then, has nothing special to do with the question whether shape perception is externalist or not. The discussion of it is a red herring.

As far as the debate between internalism and externalism goes, then, we could as well have started with the problem, alluded to above, that internalism is supposed to face that externalism does not. The problem is supposed to be that

these sensational characteristics [which form a phenomenal space for representing geometry] are supposed to be known from one's own case, it is your own experience of shape that gives you knowledge of what these sensational characteristics are. So this view has no way of explaining what it is for sensational experiences of shape to be the same or different in different observers, or for a single observer over a period of time. (p. 314)

In contrast,

The obvious way of providing an account is to move in the direction of the externalist, drawing in environmental considerations, so that sameness or difference of experience depends on the sameness or difference of the geometrical properties of the objects perceived. (p. 315)

What is the problem here? Campbell does not explain. It is unclear whether Campbell is worried about how the internalist explains what it is for two individuals both to be having a perception of, say, a 
round object, or whether his concern is with how the internalist can explain what it is for two individuals both to be having, say, a visual perception of a round object. But in neither case is it obvious what the problem is supposed to be. What is it for A and B both to have a perceptual state which represents there being a round object in front of them? It is for both A and B to have a perceptual state which represents a round object in front of them. What is it for A and $B$ both to have a visual perceptual state, as opposed to tactile, which represents there being a round object in front of them? It is for it for A and B both to have a visual perceptual state which represents there being a round object in front of them. Why is this answer not available to the internalist? Why should the fact that the internalist claims (if he does) to know what experience of shape is like from his own case raise any difficulty? ${ }^{13}$ Until more argument is forthcoming, we should conclude that there isn't even an apparent problem for the internalist in saying what it is for two individuals or an individual at two times to be having perceptual states which represents shape with the same content.

Apart from this, we should note that Campbell is assuming that any internalist about shape perception would have to appeal to a phenomenal space account of shape perception, and that any externalist account would eschew such an account. However, an internalist may very well reject a phenomenal space account of shape perception. To be an internalist, all that is needed is that one deny that the property of having an experience representing a shaped object is a relational property. Being an internalist does not carry any commitment about the relation between representations of shape and phenomenal content. Similarly, being an externalist does not require any commitment to rejecting the phenomenal space account. Thus, even if the phenomenal space account were shown to be untenable, this would not show that externalism was to be favored over internalism.

I suspect that Campbell has assumed that internalism carried a commitment to a phenomenal space account of perception of shape

\footnotetext{
${ }^{13}$ It may be that Campbell has in mind passages (esp. sections 258ff) in Wittgenstein's Philosophical Investigations that are sometimes called the private language argument, which is briefly mentioned early in the paper (p. 303). These arguments, if they are arguments, amount to little more than the assumption that we cannot recognize subjective features of our experience and identify experiences at different times as the same on the basis of memory. The thought that we can recognize something about ourselves in a way no one else can or with an authority no one else's can equal is neither a threat to the normativity of judgments about experience, nor a guarantee of infallibility.
} 
because he assumed that any internalist would want a reductive account of shape perception, and assumed that the only possible account would be a phenomenal space account. It is by no means clear that this would be the only reductionist option available to an internalist, so it is unclear even that an internalist with the commitment to give a reductive account of perceptual representation of shape would face difficulties if it were shown that the phenomenal space account could not be maintained.

Finally, in connection with these last two points, it has occurred to me that Campbell may be supposing, though he nowhere says this in the paper, that there is a special connection between the view that shape properties are categorical and the debate between internalism and externalism because he supposes that it is only if shape properties are categorical that the internalist must appeal to the phenomenal space account of shape perception, with its supposed attendant difficulties. We have seen that the assumption on which this connection is based is false because one can be an internalist, and perhaps even a reductive internalist, without adopting the phenomenal space account of shape perception. Apart from this, however, it is unclear why one would suppose that similar issues did not arise for perception of clusters of causal powers through more than one sensory modality.

To sum up, we have found no connection between questions about the nature of shape properties and the determination of the contents of representations of shaped objects. It appears that the main line of Campbell's argument is that only externalism could explain how perception of shape properties is possible if shape properties are categorical. However, on examination, the categoricity of shape properties appears to play no role in the argument. The rejection of internalism, given the categoricity of shape properties, is instead based on an objection to a phenomenal space account of shape perception. That kind of account, however, is not specially tied to internalism or externalism, nor to the categoricity or non-categoricity of the properties we perceive objects as having. Thus, the disconnection between the issues is complete. 


\section{What is the relation between externalism and the rationality of the cross-modal transfer of information, and between externalism and the question whether there are phenomenal differences between perception of shape in sight and touch?}

The argument for a connection between radical externalism and the rationality of cross-modal transfer of information is that (i) if externalism about shape perception is correct, then there is no difference in the phenomenal character of shape experience in sight and touch; and (ii) if there is no difference in the phenomenal character of shape experience in sight and touch, then "the sameness of property perceived in sight and touch is transparent to the subject"; and (iii) if the sameness of property perceived in sight and touch is transparent to the subject, then cross-modal transfer of information is rational (p. 304). (i) here, of course, is just [T2]. So a discussion of the argument for [T3] involves inter alia a discussion of [T2].

There are reasons to think that the cross-modal transfer of information is rational regardless of whether internalism or externalism is correct (see section 3 and footnote 2). But it is not clear that this argument should convince us of it independently even if we grant that externalism about shape perception is correct. We have already remarked that externalism is independent of the phenomenal space account of shape perception, and so independent of the question whether there is a difference in the phenomenal character of shape experience in sight and touch. Thus, we have no reason to believe (i), and, hence, no reason to believe [T2]. Further, it is not clear we have good reason to accept (ii) on the basis of a connection between the antecedent and consequent. Suppose that shape experience as such has no phenomenal character, and, hence, that there is no difference in the phenomenal character of shape experiences in sight and touch. Is it obvious that the content of experiences of shape should therefore be transparent to the subject? I can see that one may want to hold as a general matter than the contents of experiences are transparent, or at least accessible in consciousness, to their subject - I believe this is correct- - but this does not seem to follow from the remark that there is no phenomenal difference between perception of shape in sight and touch. In addition, if one does hold the view that the contents of experience are accessible to the subject, then it is not clear why phenomenal differences would 
prevent a subject from recognizing that he perceives the same shape by sight and touch, since, by hypothesis, the representational content of the perceptions, even if involving different phenomenal characters, are the same. Thus, it does not seem that phenomenal sameness of shape representation in sight and touch is what is crucial to question whether cross-modal transfer of information is rational.

\section{Conclusion}

I conclude that shape properties are categorical, that the crossmodal transfer of information is rational, but that Campbell has given us no reason to think that any of [T1]-[T3] is correct.

\section{REFERENCES}

Campbell, John. 1995. "Molyneux's Question". This volume, pp. 301-318. Martin, Michael. 1992. "Sight and Touch". In The Contents of Experience, ed. Tim Crane, 196-215. Cambridge: Cambridge University Press.

Davidson, Donald. 1980. "The Individuation of Events". In Essays on Actions and Events. Oxford: Oxford University Press.

Gregory, Richard L. 1990. Eye and Brain: the psychology of seeing. Princeton: Princeton University Press.

Locke, John. 1975. An Essay Concerning Human Understanding. Oxford: Oxford University Press.

O'Shaughnessy, Brian. 1989. The Sense of Touch. Australasian Journal of Philosophy 67: 37-58.

Quine, W.V. 1985. "Events and Reification". In Actions and Events: Perspectives on the Philosophy of Donald Davidson, ed. Ernest LePore and Brian McLaughlin, 162-171. Oxford: Basil Blackwell.

Shoemaker, Sydney. 1984. "Causality and Properties". In Identity, Cause, and Mind. Cambridge: Cambridge University Press.

Wittgenstein, Ludwig. 1953. Philosophical Investigations. New York: MacMillan. 